\title{
Effects of Heat Stress on Productive Performance and Some Blood Parameters in Commercial White Nicholas and Black Baladi Turkey Breeds
}

\author{
Osman, M. M ${ }^{1}$; El Bayomi KH.M ${ }^{2}$; El Nabtiti, A. A. S ${ }^{1}$ \\ and Kasem, H. H. A. ${ }^{1}$ \\ ${ }^{1}$ Department of Animal Wealth Development, Faculty of Veterinary \\ Medicine, Suez Canal University, Ismailia. \\ ${ }^{2}$ Department of Animal Wealth Development, Faculty of Veterinary \\ Medicine, Zagazig University.
}

\begin{abstract}
The present study was carried out to examine the effects of heat stress on productive performance and some blood parameters of two turkey breeds namely Commercial White Nicholas (WW) and Black Baladi (BB).

Seventy two birds (36 birds for each breed) were divided equally into three groups (12 birds for each group) as follows: group A was served as a control and reared at $24{ }^{\circ} \mathrm{C} \pm 2$, the other two groups (B and C) considered as heat stressed groups were exposed to $30{ }^{\circ} \mathrm{C}$ \pm 2 and $35^{\circ} \mathrm{C} \pm 2$, respectively. Results revealed negative impact of heat stress on productive performance parameters including live body weight, body weight gain, feed consumption and feed conversion ratio which significantly $(\mathrm{p} \leq 0.01)$ depressed in both groups (B and C). Effect of breed revealed that White Nicholas significantly $(\mathrm{p} \leq 0.01)$ improved the measured productive performance parameters in comparison with Black Baladi. inverse relationships were observed between increasing temperature and both total red blood cells count (TRBC X $10^{6}$ cells/UL) and hemoglobin concentration $(\mathrm{Hb} \mathrm{g} / \mathrm{dl})$ which significantly decreased $(\mathrm{P} \leq 0.01)$ in both heat stress groups (B followed by $\mathrm{C})$. Results of differential leucocyte count revealed significant increase $(p \leq 0.01)$ in basophils percent and lowered lymphocytes associated with high $\mathrm{H} / \mathrm{L}$ ratios (Heterophil to Lymphocyte ratio) in both $\mathrm{B}$ and $\mathrm{C}$ groups. Breed effects on TRBC, TWBC and $\mathrm{H} / \mathrm{L}$ ratio revealed significant decrease in TRBC, WBC and lymphocyte percent with significant $(p \leq 0.01)$ increase in $H / L$ ratio in Black Baladi. Corticosterone concentration shows a high significant $(\mathrm{P} \leq 0$. 01) increase while $\mathrm{T} 3$ and $\mathrm{T} 4$ showed a significant decrease $(\mathrm{P} \leq 0.01)$ in heat stress groups especially in Black breed. therefore, this study concluded that exposure of birds to high temperature $\left(30\right.$ and $\left.35^{\circ} \mathrm{C}\right)$ leads to depression of productive performance parameters, decreasing TRBC, TWBC percentages, increasing of $\mathrm{H} / \mathrm{L}$ ratio and
\end{abstract}


corticosterone concentration, and alter body temperature regulating thyroid hormones. Moreover, turkey breed had a great effect on all the above measured parameter as the Black Baladi breed was more susceptible to stress.

Key words: Turkey, Heat Stress, White Nicholas, Black Baladi, productive performance

\section{Introduction}

The consumption of turkey meat has been grown recently in Egypt, as our population continues to grow and become more urbanized, so it is necessary to continue the trend of intensive poultry production (ElLaithy, 2003). Poultry seems to be sensitive to temperature-associated environmental challenges, especially heat stress so it is crucial to understand and control environmental conditions for successful poultry production and welfare (Lara and Rostagno, 2013).

The most important inhibiting factors to poultry production in hot regions are the high environmental temperatures (Melesse et al, 2011) thus, the effect of high environmental temperature on the productive performance of different poultry species including turkeys had been investigated by many researchers who reported deleterious effects of high environmental temperatures which is called heat stress on productive performance such as depresses body weight and a general suppression of feed intake (Scott and Balnave, 1988 and Mckee and Sams, 1997). Heat stress (HS) is considered as one of the most challenging environmental conditions affecting poultry production, that causes negative effects including decreased growth performance, high mortality due to a cascade of irreversible thermoregulatory events and large economic losses in poultry (Yahav, 2000; Gu et al, 2012; Kamboh et al, 2013; Sahin et al, 2013). At the same time High environmental temperatures require many physiological (Star et al, 2008 and 2009) and immunological adaptations of birds.

Exposure of birds to high temperature leads to a decrease in the circulating red blood cells, total and differential leucocytic count (Vecerek et al, 2002) and hemoglobin concentration (Comito et al, 2007) with an increase in the $\mathrm{H} / \mathrm{L}$ ratio (Maxwell et al, 1992).

Heat stress impairs the activity of the neuroendocrine system of poultry, leading to a modified neuronedocrine profile by activation of the hypothalamic-pituitaryadrenal (HPA) axis, and elevated plasma corticosterone concentrations (Garriga et al, 2006 and Quinteiro-Filho et al, 2012). In addition to balance alteration of body temperature regulating thyroid hormones, triiodothyronine (T3) and thyroxine (T4) either by 
decreasing T3 and or decreasing T4 (Deeb, and Cahaner, 2002), (Elnagar et al, 2010) or no alteration (Mack et al, 2013) of T4.

Therefore, this study was designed in order to investigate the effects of heat stress on the live bird performance parameters including feed consumption, live body weight, feed conversion ratio (FCR), and live body weight gain in two turkey breeds namely Egyptian Black Baladi breed (B.B) and Commercial White Nicholas Breed (W.W) . Furthermore, blood analysis was carried out to measure total red blood cells count (TRBC), hemoglobin concentration $(\mathrm{Hb})$, total and differential leucocytic count, heterophile to lymphocyte ratio $(\mathrm{H} / \mathrm{L}$ ratio), $\mathrm{T} 3$ and $\mathrm{T} 4$ hormone, and corticosterone concentration.

\section{Materials and Methods}

\section{Experimental birds:}

Seventy two male turkey poults (8 weeks old) of two Egyptian turkey breeds [36 Black Baladi breed (BB), and 36 White Nicholas breed (WW)] with body weight ranged between 800-1100 $\mathrm{g}$ and 2700$2900 \mathrm{~g}$ in (BB) and (WW) breed, respectively were used in this study . The experimental birds were divided into 3 groups with 3 replicates which allocated according to the heat exposure as following (Skrbic et al, 2009): control group (A), birds were reared at $24^{\circ} \mathrm{C} \pm 2$ as recommended by Osman et al (1989), heat treated groups (B and
C) were allowed by thermostatically controlled electric heaters for providing the needed temperatures $30^{\circ} \mathrm{C} \pm 2$ and $35^{\circ} \mathrm{C} \pm 2$. Each bird was provided with a floor space of 0.25 square meters (Sainsbury, 2000) with adequate light intensity reaching 30.8 lux. The lighting period was 23 hours a day as recommended by Mercia (1986).

Thermostatically controlled electric heaters were used to provide the needed temperature in heat stress experiment, and electric fans for the air circulation. Grower and finisher diets were formulated according to (NRC, 1994). Feed and water were offered ad-libitum in cleaned feeders and drinkers, and the birds were fed basal diet. As Waibel et al (2000) addressed presently balance feed of turkey is not commercially available, the ration were processed manually, the ingredients and chemical composition of the diets are illustrated in Table 1.

II. Measurement of productive performance parameters:

The following productive performance parameters were recorded throughout the experiment:

(1) Live Body Weight was individually recorded every two weeks (Richards, 1959).

(2) Body Weight Gain between two successive weeks was individually calculated according to the following formula: Weight gain $(\mathrm{gm})=\mathrm{W} 2-\mathrm{W} 1$ (Where W1 and W2 $=$ weight of individual at two successive weeks). 
(3) Feed intake (FI):

Feed consumed by all birds in each treatment was recorded; it was then averaged and expressed in gm per bird per day in each experimental group.

Feed intake (FI) (gram / bird $/$ period) $=$ Amount of feed consumed of each replicate / Number of chicks consumed feed, (Brody, 1968).

(4) Feed Conversion Ratio (FCR): It was calculated for each group during different periods of the experiment and at the end of the experiment as follows: F.C.R = Feed consumption (g)/ Weight gain (g) according to Euribrid (1982).

\section{Blood parameters:}

Blood samples were collected from the wing vein from all turkeys at the beginning of the experiment (8 weeks old) then at $18^{\text {th }}, 20^{\text {th }}$, and $24^{\text {th }}$ weeks. Blood collected 4 times only to avoid a macrocytic hypochromic anemia (Christie, 1978) caused by repeated bleeding. Blood analysis carried out to measure the following parameters:

1. Total Red Blood Cells count (TRBC) count was estimated using hemocytometer, Hemoglobin concentration $(\mathrm{Hb}) \mathrm{g} / \mathrm{dl}$ was measured by colorimeter using cyanmethaemoglobin method.

2. Total Leukocytic Count (TWBC) $* 10^{3}$ cells $/ \mathrm{mm}^{3}$ and Diffrential Leukocytic Count including Lymphocyte\%, Monocyte\%, Eosinophil\%, Heterophil (Neutrophile) \%, Basophil\% were counted using the haemocytometer method . Heterophil to Lymphocyte Ratio (H/L ratio) were calculated according to Gross and Siegel (1983) by dividing the number of heterophils in $1 \mathrm{~mL}$ of peripheral blood by the number of lymphocytes.

3. Serum hormones: (T3, T4, corticosterone concentration)

The serum hormone concentration free triiodothyronine (fT3) and free thyroxine (fT4) concentrations was determined by the radioimmunological assay using a diagnostic kit Code: B-R-A-H-M-S (Roche Diagnostics Gmbh, Mannheim, Germany) and the Elecsys 1010 analyzer (Instrument Center AG, Rotkreuz, Switzerland) as described in the manufacturer's manuals.

Corticosterone concentration $\mathrm{ng} / \mathrm{ml}$ was determined by the radioimmunological assay for corticosterone analysis, blood samples were kept at room temperature until the serum separated. Then, the serum samples were immediately assayed for corticosterone concentration with a commercial ELISA kit (Assay Designs, catalogue no: 900-097) as described in the manufacturer's manuals.

\section{Statistical analysis:}

Data were analyzed statistically using general linear procedure of analysis of variance (ANOVA). Factorial experiments were included for the analyses of main and interaction effects for both heat stress and stocking density, 
followed by means separation for significance according to Duncan's Multiple Range Test (DMRT) using (SPSS version 16) software for main effects significance, while MSTAT software was used for mean separation and interactions. Results considered significant at ( $\mathrm{P}$ $\leq 0.05)$.

Statistical models: The following model was assumed to analyze the factors affecting the characteristics studied in the heat stress experiment:

$Y_{i j k l}=\mu+H_{i}+B_{j}+A_{k}+H_{i j}+e_{i j k l}$ Where,
$\mathrm{Y}_{\mathrm{ijkl}}=$ is the observation of the dependent variable.

$\mu \quad=$ is an overall mean common to all animals in the experiment.

$\mathrm{H}_{\mathrm{i}} \quad=$ is the effect due to heat stress, $\mathrm{i}=1,2,3$ (where, $1=$ control group, $2=30$ degree group, $3=35$ degree group).

$B_{j} \quad=$ is the effect of breed, $j=1,2$ (where, 1= Black Baladi turkey, 2 = White Nicholas turkey).

$A_{k}=$ is the effect of age, $k=1,2,3$, (where, $1=8$ weeks, $2=10$ weeks, $3=12$ weeks, ...).

$\mathrm{HB}_{\mathrm{ij}}=$ is an interaction effect of heat stress and breed.

$\mathrm{e}_{\mathrm{ijk} \mathrm{l}}=$ is the residual effect.

Table (1): The Composition and Nutritive Value of the grower and finisher Turkey Diets.

\begin{tabular}{|c|c|c|c|c|c|}
\hline Inomolionto/ & $\begin{array}{c}\text { Grower } \\
\text { diet }\end{array}$ & $\begin{array}{c}\text { Finisher } \\
\text { diet }\end{array}$ & $\begin{array}{c}\text { Calculated } \\
\text { analysis }^{2}\end{array}$ & $\begin{array}{c}\text { Grower } \\
\text { diet }\end{array}$ & $\begin{array}{c}\text { Finisher } \\
\text { diet }\end{array}$ \\
\hline Ingredient\% & $\begin{array}{c}\text { 9-16 } \\
\text { weeks }\end{array}$ & $\begin{array}{l}17-24 \\
\text { weeks }\end{array}$ & \multirow{2}{*}{$\mathrm{ME}(\mathrm{kcal} / \mathrm{kg})$} & $\begin{array}{c}\text { 9-16 } \\
\text { weeks }\end{array}$ & $\begin{array}{l}17-24 \\
\text { weeks }\end{array}$ \\
\hline $\begin{array}{c}\text { Ground yellow } \\
\text { corn }\end{array}$ & 56.5 & 67.7 & & 3000 & 3200 \\
\hline $\begin{array}{c}\text { Soybean meal } \\
(44 \%)\end{array}$ & 35 & 25.7 & ср\% & 22 & 16 \\
\hline $\begin{array}{c}\text { Herring fish meal } \\
(72 \%)\end{array}$ & 3 & - & $\mathrm{ca} \%$ & 0.85 & 0.65 \\
\hline Corn oil & 2.7 & 4.2 & $\mathrm{ph} \%$ & 0.42 & 0.32 \\
\hline $\begin{array}{l}\text { Di-calcium - } \\
\text { phosphate }\end{array}$ & 1.3 & 1.1 & $\mathrm{EE} \%$ & 5.45 & 6.92 \\
\hline Limestone & 0.79 & 0.7 & $\mathrm{CF} \%$ & 3.87 & 3.43 \\
\hline Methionine & 0.06 & - & Methionine $\%$ & 0.45 & 0.28 \\
\hline Lysine & 0.05 & - & $\begin{array}{c}\text { Methionine }+ \\
\text { cystine } \%\end{array}$ & 0.8 & 0.57 \\
\hline Common salt & 0.3 & 0.3 & Lysine \% & 1.3 & 0.87 \\
\hline $\begin{array}{l}\text { Vit.and Min. } \\
\text { mixture }^{1}\end{array}$ & 0.3 & 0.3 & $\operatorname{Niacin}^{3}(g) \%$ & 2.53 & 2.28 \\
\hline Total & 100 & 100 & $\operatorname{Biotin}^{4}(\mathrm{~g}) \%$ & 0.02 & 0.01 \\
\hline
\end{tabular}

${ }^{I}$ Vit. And Min. Mixture: (Multi- mix) the Egyptian - Holland Co. ${ }^{\circledR}$. Each gram of vitamin premix contains: Vit. A 20000 I.U, Vit.D3 2000 I.U, Vit. E $400 \mathrm{I} . \mathrm{U}$, Vit. $\mathrm{K} 2 \mathrm{mg}$, niacin $20 \mathrm{mg}$, riboflavin $4.5 \mathrm{mg}$, pyridoxine $3 \mathrm{mg}$, B12 
$0.013 \mathrm{mg}$ and choline chloride 100mg. Each gram of the trace mineral premix contains $\mathrm{Mn}$. 40mg, Zn $45 \mathrm{mg}$, copper $3 \mathrm{mg}$, iodine $0.3 \mathrm{mg}$, selenium $0.1 \mathrm{mg}$ and iron $30 \mathrm{mg}$.

${ }^{2}$ Calculated according to National Research Council (1994).

${ }^{3}$ Niacin calculated from yellow corn, soybean meal and fish meal acc. to National Research Council (1994).

${ }^{4}$ Biotin calculated from yellow corn, soybean meal and fish meal acc. to National Research Council (1994).

\section{Results}

Effects of heat stress and breed on productive performance parameters were illustrated in (table 2). In one hand, results of the effect of heat stress revealed an inverse relationship between live body weight and higher temperature levels, whereas, highly significant decrease $(p \leq 0.01)$ was observed in both heat-treated groups (B and C) compared with the control group. The same pattern was reported in body weight gain and feed intake. Results of FCR found to be improved in Group A, which significantly differ from other groups (B and $C$ ). On the other hand, effect of breed revealed that White Nicholas significantly $(\mathrm{p} \leq$ 0.01 ) improved the measured productive performance parameters in comparison with Black Baladi. Influence and interaction of the breed on the heat treated group was tabulated in (Table 2) which revealed that White Nicholas breed had highly significant interaction $(p \leq 0.01)$ in the all heat treated groups (A, B and C) when compared to Black Baladi in the productive performance parameters.
Table 2 shows that the age had a high significant $(\mathrm{P} \leq 0.01)$ effect on turkey live body weight as in lowest age in the experiment ( 8 weeks) has been the lowest weight (2194.53g) and the last age in the experiment (24 weeks) has been the highest weight $(7671.57 \mathrm{~g})$. But the obtained results had shown the profit per bird was at 16 weeks of rearing, followed by 20 weeks and was found maximum of 24 weeks, and the turkey farm owners in Egypt are rearing turkeys till (20-21 weeks). Age effect was highly significant $(p \leq 0.001)$ on weight gain of bird as the period of (8-10 weeks) had the lowest weight gain $(550.35 \mathrm{~g})$, while the period of (22-24 weeks) was the highest weight gain (1028.26g). The age had a highly significant $(\mathrm{p} \leq 0$. 001) effect on food consumed by turkeys as the highest value was in the period from $22-24$ weeks (4423.57) and the lowest value was in the 10-12 week period (1824.68g).

Age had a highly significant effect on turkey FCR. The period from 1416 weeks showed highest FCR (5.01) and in the period from 8-10 weeks FCR was (4.24) 
The effect of heat stress on TRBC $\mathrm{X} 10^{6}$ cells/UL and $\mathrm{Hb}$ g/dl and $\mathrm{H} / \mathrm{L}$ ratio data were illustrated in Figures 1 and 2. An inverse relationship was observed between increasing temperature and the measured blood parameters, which could be expressed in the form of significant decrease $(\mathrm{P} \leq 0.01)$ in both heat stress groups (B followed by $C$ ) which observed when compared to control in TRBC $\times 10^{6}$ cells/UL and $\mathrm{Hb} \mathrm{g} / \mathrm{dl}$. Effect of breed on the measured parameters (Figures 1 and 2) can be concluded as follows, White Nicholas showed highly significant increases $(P \leq 0.01)$ in TRBC $X 10^{6}$ cells and $\mathrm{Hb} \mathrm{g} / \mathrm{dl}$ at $24^{\text {th }}$ week age. Interaction between groups and breeds had a high significance $(P \leq 0.01)$ values with the control group with a white breed showed the highest values in TRBC $\mathrm{X} 10^{6}$ cells/UL while, the $35^{\circ} \mathrm{C}$ group with black breed obtained the lowest (Figures 1 and 2).

Data referring to differential leukocyte counts are presented in Table 3 showed that TWBC X $10^{3}$ cells $/ \mathrm{mm}^{3}$ with especial reference to lymphocyte \% showed a significant decrease $(\mathrm{P} \leq 0$. 01) in group $\mathrm{C}$ followed by group B as compared with control. Different patterns were observed in monastic, heterophils and basophils \%, which showed significant increase $(\mathrm{P} \leq 0$. 01 ) in both groups B and C. On the other hand, $\mathrm{H} / \mathrm{L}$ ratio showed a significant increase $(\mathrm{P} \leq 0$. 01) with the highest level in group $\mathrm{C}$ followed by group B in comparison with control. Concerning breed effect, TWBC $\times 10^{3}$ cells $/ \mathrm{mm}^{3}$, with lymphocyte and Eosinophils \% showed a significant decrease $(\mathrm{P} \leq 0$. 01) in Black Baladi, while monocyte, heterophils and basophils \% with $\mathrm{H} / \mathrm{L}$ ratio which showed a significant increase $(\mathrm{P} \leq 0$. 01). At the same time age of birds did not show any significant change in TWBC X $10^{3}$ cells $/ \mathrm{mm}^{3}$ in turn it was reflected on $\mathrm{H} / \mathrm{L}$ ratio.

Interaction between groups and breeds represented high significance $(\mathrm{P} \leq 0.01)$ values where the control group with a white breed showed the highest values in TWBC $\times 10^{3}$ cells $/ \mathrm{mm}^{3}$ and lowest values in $\mathrm{H} / \mathrm{L}$ while, the $35^{\circ} \mathrm{C}$ group with black breed obtained the lowest and highest value in (TWBC $\times 10^{3}$ cells $/ \mathrm{mm}^{3}$,) and $\mathrm{H} / \mathrm{L}$ ratio, respectively (Table 3 )

Data reported in (Table 4) showed that corticosterone concentration had a high significant $(P \leq 0.01)$ effect between heat stress groups. Age effect was significant in corticosterone concentration $(\mathrm{P} \leq 0$. 056). Interaction between groups and breed had high significant values $(\mathrm{P} \leq 0$. 001) whereas, the control group with black breed showed the lowest value and the $35^{\circ} \mathrm{C}$ group with a white breed obtained the highest value.

T3 and T4 showed a highly significant difference between heat stress groups $(P \leq 0.01)$ in the form of significant decrease $(P \leq 0.01)$ in heat stress groups among turkey breed with a highly significant 
decrease in-group $\mathrm{C}$ and in Black Baladi. An increase in T3 and T4 were observed with the older age $\left(24^{\text {th }}\right.$ week $)$ which were significantly differ in T3 and nonsignificant in $\mathrm{T} 4$. Interaction between groups and breed reported that the control group with a white breed showed the highest value and the $35^{\circ} \mathrm{C}$ group with black breed obtained the lowest value (Table 4).

Table (2): Effects of heat stress, breed, their interaction and age on productive performance parameters (Means \pm Standard Errors).

\begin{tabular}{|c|c|c|c|c|c|}
\hline \multicolumn{2}{|c|}{$\begin{array}{l}\text { Main and interaction } \\
\text { Effects }\end{array}$} & Live weight (gm ) & $\begin{array}{l}\text { Body weight gain } \\
(+\mathrm{L}: \mathrm{Ngm})\end{array}$ & Feed Intake (gm ) & $\begin{array}{l}\text { Feed } \\
\text { Conversion } \\
\text { Ratio (FCR) }\end{array}$ \\
\hline \multicolumn{6}{|c|}{ Heat stress (observation No. $=216$ for each group) } \\
\hline \multicolumn{2}{|l|}{ Group A } & $5250.78^{\mathrm{a}} \pm 229.61$ & $1599.21^{\mathrm{a}} \pm 157.58$ & $6134.46^{\mathrm{a}} \pm 581.90$ & $4.24^{\mathrm{c}} \pm 0.08$ \\
\hline \multicolumn{2}{|l|}{ Group B } & $4415.43^{\mathrm{b}} \pm 172.42$ & $1123.57^{\mathrm{b}} \pm 107.07$ & $4898.12^{\mathrm{b}} \pm 442.62$ & $4.88^{\mathrm{b}} \pm 0.11$ \\
\hline \multicolumn{2}{|l|}{ Group C } & $4215.16^{\mathrm{c}} \pm 151.57$ & $925.81^{\mathrm{c}} \pm 90.36$ & $4489.07^{\mathrm{c}} \pm 405.01$ & $5.44^{\mathrm{a}} \pm 0.12$ \\
\hline \multicolumn{6}{|c|}{ Breed (observation No. $=324$ for each breed) } \\
\hline \multicolumn{2}{|c|}{ White Nicholas } & $6528.75^{\mathrm{a}} \pm 148.72$ & $1722.26^{\mathrm{a}} \pm 126.31$ & $6738.85^{\mathrm{a}} \pm 483.86$ & $3.94^{\mathrm{b}} \pm 0.03$ \\
\hline \multicolumn{2}{|c|}{ Black Baladi } & $2725.50^{\mathrm{b}} \pm 59.31$ & $710.14^{\mathrm{b}} \pm 51.80$ & $3608.92^{\mathrm{b}} \pm 252.45$ & $5.77^{\mathrm{a}} \pm 0.10$ \\
\hline \multicolumn{6}{|c|}{ Breed X Heat stress (observation No. $=108$ for each treatment) } \\
\hline \multirow{2}{*}{ Group A } & $\begin{array}{c}\text { White } \\
\text { Nicholas }\end{array}$ & $7473.08^{\mathrm{a}} \pm 323.38$ & $2317.02^{\mathrm{a}} \pm 279.81$ & $8469.55^{\mathrm{a}} \pm 1023.52$ & $3.59^{\mathrm{f}} \pm 0.03$ \\
\hline & Black Baladi & $3028.48^{\mathrm{d}} \pm 122.38$ & $881.41^{\mathrm{d}} \pm 108.90$ & $3799.37^{\mathrm{d}} \pm 459.62$ & $4.88^{\mathrm{c}} \pm 0.15$ \\
\hline \multirow{2}{*}{ Group B } & $\begin{array}{l}\text { White } \\
\text { Nicholas }\end{array}$ & $6242.47^{\mathrm{b}} \pm 218.43$ & $1562.26^{\mathrm{b}} \pm 188.02$ & $6235.26^{\mathrm{b}} \pm 751.65$ & $3.93^{\mathrm{e}} \pm 0.04$ \\
\hline & Black Baladi & $2588.40^{\mathrm{e}} \pm 96.70$ & $684.89^{\mathrm{e}} \pm 84.41$ & $3560.98^{\mathrm{e}} \pm 434.64$ & $5.84^{\mathrm{b}} \pm 0.17$ \\
\hline \multirow{2}{*}{ Group C } & $\begin{array}{l}\text { White } \\
\text { Nicholas }\end{array}$ & $5870.70^{\mathrm{c}} \pm 186.70$ & $1287.50^{\mathrm{c}} \pm 159.80$ & $5511.74^{\mathrm{c}} \pm 680.61$ & $4.29^{\mathrm{d}} \pm 0.06$ \\
\hline & Black Baladi & $2559.62^{\mathrm{e}} \pm 78.99$ & $564.13^{\mathrm{f}} \pm 69.54$ & $3466.41^{\mathrm{e}} \pm 420.02$ & $6.59^{\mathrm{a}} \pm 0.17$ \\
\hline \multicolumn{6}{|c|}{ Age (observation No. $=72$ for each week) } \\
\hline \multicolumn{2}{|l|}{$8^{\text {th }}$ week } & $2194.53^{\mathrm{i}} \pm 91.01$ & $550.35^{\mathrm{ef}} \pm 41.41$ & $1944.32^{\mathrm{e}} \pm 113.63$ & $4.24^{\mathrm{b}} \pm 0.18$ \\
\hline \multicolumn{2}{|l|}{$10^{\text {th }}$ week } & $2744.87^{\mathrm{h}} \pm 131.72$ & $505.74^{\mathrm{f}} \pm 40.27$ & $1824.68^{\mathrm{e}} \pm 89.71$ & $5.32^{\mathrm{a}} \pm 0.27$ \\
\hline \multicolumn{2}{|l|}{$12^{\text {th }}$ week } & $3250.60^{\mathrm{g}} \pm 170.89$ & $591.50^{\mathrm{de}} \pm 28.58$ & $2569.26^{\mathrm{d}} \pm 102.82$ & $4.55^{\mathrm{b}} \pm 0.14$ \\
\hline \multicolumn{2}{|l|}{$14^{\text {th }}$ week } & $3842.11^{\mathrm{f}} \pm 194.78$ & $619.03^{\mathrm{de}} \pm 35.71$ & $2761.79^{\mathrm{d}} \pm 103.26$ & $5.01^{\mathrm{a}} \pm 0.20$ \\
\hline \multicolumn{2}{|l|}{$16^{\text {th }}$ week } & $4461.14^{\mathrm{e}} \pm 225.26$ & $646.42^{\mathrm{d}} \pm 45.97$ & $2817.97^{\mathrm{d}} \pm 130.67$ & $5.17^{\mathrm{a}} \pm 0.24$ \\
\hline \multicolumn{2}{|l|}{$18^{\text {th }}$ week } & $5114.33^{\mathrm{d}} \pm 266.20$ & $726.08^{c} \pm 49.33$ & $3266.35^{\mathrm{c}} \pm 143.09$ & $5.11^{\mathrm{a}} \pm 0.18$ \\
\hline \multicolumn{2}{|l|}{$20^{\text {th }}$ week } & $5840.42^{\mathrm{c}} \pm 308.81$ & $802.89^{\mathrm{c}} \pm 57.60$ & $3677.72^{\mathrm{b}} \pm 196.15$ & $5.17^{\mathrm{a}} \pm 0.19$ \\
\hline \multicolumn{2}{|l|}{$22^{\text {nd }}$ week } & $6524.56^{\mathrm{b}} \pm 365.63$ & $1028.26^{\mathrm{b}} \pm 57.52$ & $4423.57^{\mathrm{a}} \pm 181.53$ & $4.56^{\mathrm{b}} \pm 0.14$ \\
\hline \multicolumn{2}{|l|}{$24^{\text {th }}$ week } & $7671.57^{\mathrm{a}} \pm 406.76$ & $5475.53^{\mathrm{a}} \pm 327.39$ & $23279.31^{\mathrm{a}} \pm 994.29$ & $4.55^{\mathrm{b}} \pm 0.102$ \\
\hline
\end{tabular}

Differences between means within column having different superscripts are highly statistically significant $(p \leq 0.01)$

$A=$ birds reared at $24^{\circ} C \pm 2, B=$ birds exposed to heat stress at $30^{\circ} C \pm$ 2, $C=$ birds exposed to heat stress at $35^{\circ} \mathrm{C} \pm 2$ 


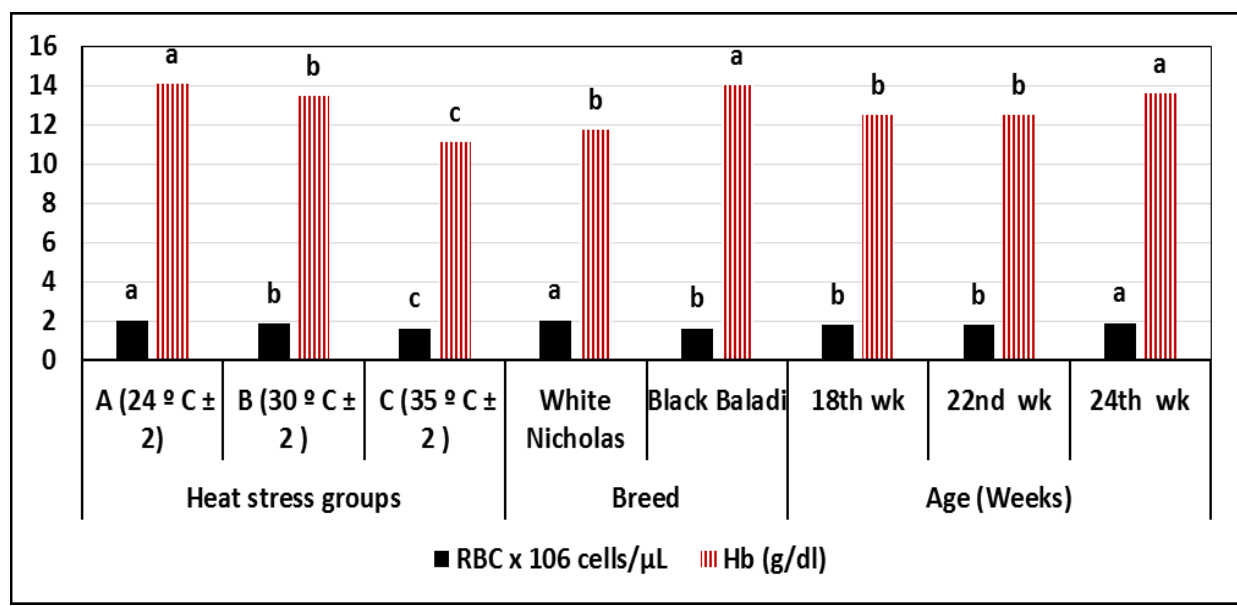

Figure1: Effects of heat stress breed and age of bird on Total Red Blood Cells (TRBC) count and hemoglobin (Hb) concentration

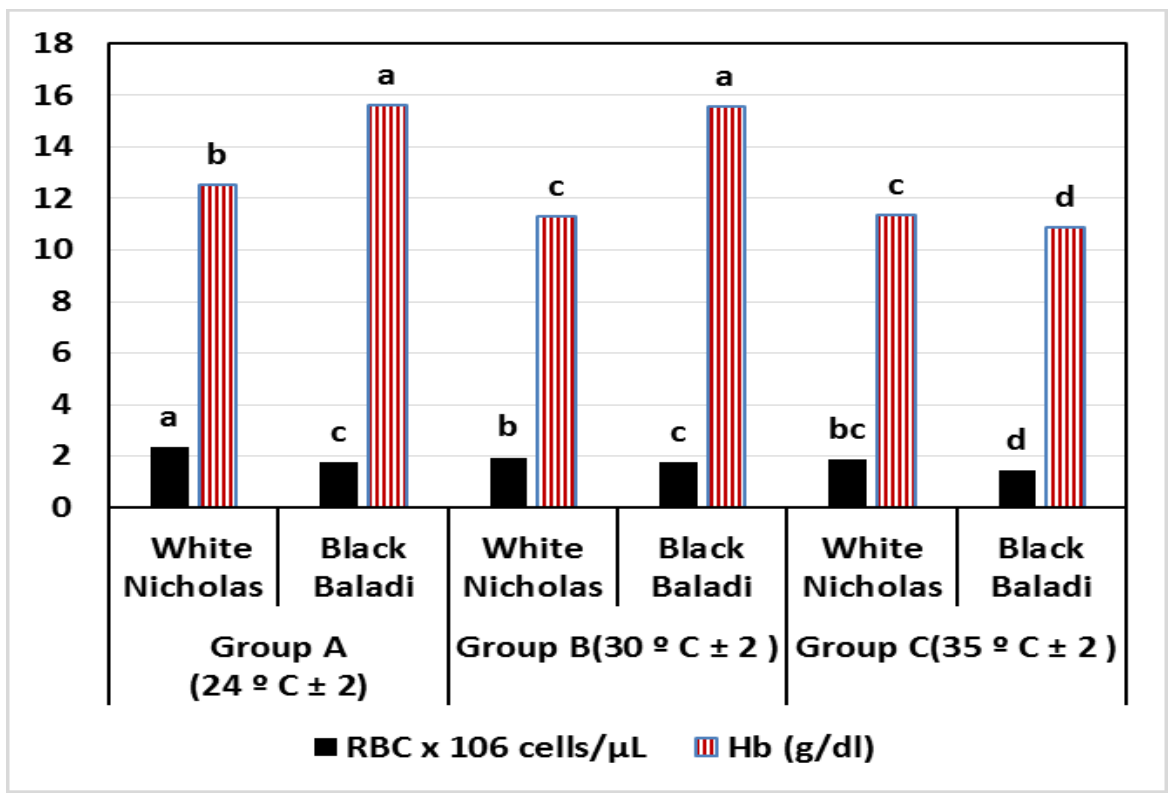

Figure2: Effects of heat stress and breed interaction on $R B C$ and $H b$ 
Table 3: Effects of heat stress, breed, age of bird and their interaction on total and differential leukocyte count (Means \pm Standard Errors):

\begin{tabular}{|c|c|c|c|c|c|c|c|c|}
\hline \multicolumn{2}{|c|}{ Effects } & $\begin{array}{l}\mathrm{WBC} \times 10^{3} \\
\text { cells } / \mathbf{m m}^{3}\end{array}$ & $\begin{array}{c}\text { Lymphocyte } \\
\%\end{array}$ & $\begin{array}{c}\text { Monocyte } \\
\%\end{array}$ & $\begin{array}{c}\text { Heterophils } \\
\%\end{array}$ & $\begin{array}{c}\text { Basophils } \\
\%\end{array}$ & $\begin{array}{c}\text { Eosinophils } \\
\%\end{array}$ & H.L ratio \\
\hline \multicolumn{9}{|c|}{ Heat stress (observation No. $=72$ for each group) } \\
\hline \multicolumn{2}{|l|}{ Group A } & $30.06^{\mathrm{a}} \pm 0.64$ & $65.87^{\mathrm{a}} \pm 0.84$ & $3.25^{\mathrm{c}} \pm 0.04$ & $24.79^{\mathrm{c}} \pm 0.81$ & $3.93^{\mathrm{c}} \pm 0.17$ & $2.20^{\mathrm{a}} \pm 0.17$ & $0.38^{\mathrm{c}} \pm 0.01$ \\
\hline \multicolumn{2}{|l|}{ Group B } & $28.95^{\mathrm{b}} \pm 0.62$ & $58.93^{\mathrm{b}} \pm 0.04$ & $3.64^{\mathrm{b}} \pm 0.02$ & $31.6^{\mathrm{b}} \pm 0.03$ & $5.08^{\mathrm{b}} \pm 0.02$ & $0.89^{c} \pm 0.02$ & $0.54^{\mathrm{b}} \pm 0.004$ \\
\hline \multicolumn{2}{|l|}{ Group C } & $27.45^{\mathrm{c}} \pm 0.52$ & $55.04^{\mathfrak{c}} \pm 0.42$ & $4.14^{\mathrm{a}} \pm 0.08$ & $34.13^{\mathrm{a}} \pm 0.3$ & $5.66^{\mathrm{a}} \pm 0.08$ & $1.46^{\mathrm{b}} \pm 0.06$ & $0.64^{\mathrm{a}} \pm 0.008$ \\
\hline \multicolumn{9}{|c|}{ Breed (observation No. $=324$ for each breed) } \\
\hline \multicolumn{2}{|c|}{ White Nicholas } & $33.34^{\mathrm{a}} \pm 0.15$ & $63.09^{\mathrm{a}} \pm 0.68$ & $3.41^{\mathrm{b}} \pm 0.04$ & $27.37^{\mathrm{b}} \pm 0.65$ & $4.17^{\mathrm{b}} \pm 0.12$ & $1.96^{\mathrm{a}} \pm 0.12$ & $0.47^{\mathrm{b}} \pm 0.01$ \\
\hline \multicolumn{2}{|c|}{ Black Baladi } & $24.33^{\mathrm{b}} \pm 0.30$ & $56.73^{\mathrm{b}} \pm 0.34$ & $3.95^{\mathrm{a}} \pm 0.06$ & $32.97^{\mathrm{a}} \pm 0.23$ & $5.61^{\mathrm{a}} \pm 0.05$ & $1.08^{\mathrm{b}} \pm 0.05$ & $0.57^{\mathrm{a}} \pm 0.02$ \\
\hline \multicolumn{9}{|c|}{ Age (observation No. $=72$ for each age) } \\
\hline \multicolumn{2}{|l|}{$18^{\text {th }}$ week } & $29.01^{\mathrm{a}} \pm 0.58$ & $59.92^{\mathrm{a}} \pm 0.76$ & $3.69^{\mathrm{a}} \pm 0.07$ & $30.19^{\mathrm{a}} \pm 0.68$ & $4.88^{\mathrm{a}} \pm 0.14$ & $1.50^{\mathrm{a}} \pm 0.12$ & $0.52^{\mathrm{a}} \pm 0.01$ \\
\hline \multicolumn{2}{|l|}{$22^{\text {nd }}$ week } & $28.99^{\mathrm{a}} \pm 0.58$ & $59.92^{\mathrm{a}} \pm 0.76$ & $3.69^{\mathrm{a}} \pm 0.07$ & $30.19^{\mathrm{a}} \pm 0.68$ & $4.88^{\mathrm{a}} \pm 0.14$ & $1.50^{\mathrm{a}} \pm 0.12$ & $0.52^{\mathrm{a}} \pm 0.01$ \\
\hline \multicolumn{2}{|l|}{$24^{\text {th }}$ week } & $28.46^{\mathrm{a}} \pm 0.66$ & $59.90^{\mathrm{a}} \pm 0.77$ & $3.66^{\mathrm{a}} \pm 0.07$ & $30.14^{\mathrm{a}} \pm 0.69$ & $4.91^{\mathrm{a}} \pm 0.14$ & $1.55^{\mathrm{a}} \pm 0.13$ & $0.52^{\mathrm{a}} \pm 0.02$ \\
\hline \multicolumn{9}{|c|}{ Breed $X$ Heat stress (observation No. $=36$ for each treatment) } \\
\hline \multirow{2}{*}{ Group A } & $\begin{array}{l}\text { White } \\
\text { Nicholas }\end{array}$ & $35.04^{\mathrm{a}} \pm 0.05$ & $72.64^{\mathrm{a}} \pm 0.50$ & $2.87^{\mathrm{c}} \pm 0.01$ & $18.22^{\mathrm{d}} \pm 0.42$ & $2.60^{\mathrm{d}} \pm 0.15$ & $3.65^{\mathrm{a}} \pm 0.006$ & $0.26^{\mathrm{d}} \pm 0.008$ \\
\hline & Black Baladi & $25.08^{\mathrm{d}} \pm 0,52$ & $59.10^{\mathrm{b}} \pm 0.05$ & $3.63^{\mathrm{b}} \pm 0.01$ & $31.35^{\mathrm{c}} \pm 0.03$ & $5.25^{\mathrm{b}} \pm 0.02$ & $0.74^{\mathrm{e}} \pm 0.020$ & $0.51^{\mathrm{c}} \pm 0.005$ \\
\hline \multirow{2}{*}{ Group B } & $\begin{array}{l}\text { White } \\
\text { Nicholas }\end{array}$ & $33.59^{\mathrm{b}} \pm 0.09$ & $58.53^{\mathrm{bc}} \pm 0.03$ & $3.67^{\mathrm{b}} \pm 0.03$ & $31.86^{\mathrm{bc}} \pm 0.01$ & $4.90^{\mathrm{c}} \pm 0.01$ & $1.04^{\mathrm{d}} \pm 0.008$ & $0.57^{\mathrm{b}} \pm 0.003$ \\
\hline & Black Baladi & $24.33^{\mathrm{de}} \pm 0.56$ & $59.12^{\mathrm{b}} \pm 0.04$ & $3.62^{\mathrm{b}} \pm 0.01$ & $31.34^{\mathrm{c}} \pm 0.02$ & $5.27^{\mathrm{b}} \pm 0.02$ & $0.73^{\mathrm{e}} \pm 0.02$ & $0.51^{\mathrm{c}} \pm 0.005$ \\
\hline \multirow{2}{*}{ Group C } & $\begin{array}{l}\text { White } \\
\text { Nicholas }\end{array}$ & $31.37^{\mathrm{c}} \pm 0.04$ & $58.10^{\mathrm{c}} \pm 0.30$ & $3.68^{\mathrm{b}} \pm 0.06$ & $32.03^{\mathrm{b}} \pm 0.30$ & $5.01^{\mathrm{c}} \pm 0.06$ & $1.18^{\mathrm{c}} \pm 0.08$ & $0.58^{\mathrm{b}} \pm 0.008$ \\
\hline & Black Baladi & $23.52^{\mathrm{e}} \pm 0.46$ & $51.98^{\mathrm{d}} \pm 0.30$ & $4.59^{\mathrm{a}} \pm 0.11$ & $36.23^{\mathrm{a}} \pm 0.13$ & $6.32^{\mathrm{a}} \pm 0.05$ & $1.75^{\mathrm{b}} \pm 0.08$ & $0.70^{\mathrm{a}} \pm 0.003$ \\
\hline
\end{tabular}

Differences between means within column having different superscripts arehighly statistically significant $(p \leq 0.01)$

$A=$ birds reared at $24^{\circ} C \pm 2 \quad B=$ birds exposed to heat stress at $30^{\circ} \mathrm{C} \pm 2 \quad \mathrm{C}=$ birds exposed to heat stress at $35^{\circ} \mathrm{C} \pm 2$ 
Table 4: Effects of heat stress, breed, age of bird and their interaction on serum hormones (Corticosterone, T3 and T4) (Means \pm Standard Errors):

\begin{tabular}{|c|c|c|c|c|}
\hline \multicolumn{2}{|c|}{ Main and interaction Effects } & $\begin{array}{c}\text { Corticosterone } \\
(\mathrm{ng} / \mathrm{ml})\end{array}$ & $\begin{array}{c}\text { T3 } \\
\text { (ng/ml) }\end{array}$ & T4 (ng/ml) \\
\hline \multirow{3}{*}{$\begin{array}{l}\text { Heat stress } \\
\text { (observation No. }=72 \text { for } \\
\text { each group) }\end{array}$} & Group A & $3.72^{b} \pm 0.10$ & $1.17^{\mathrm{a}} \pm 0.05$ & $16.77^{\mathrm{a}} \pm 0.18$ \\
\hline & Group B & $3.30^{\mathrm{c}} \pm 0.11$ & $0.79^{\mathrm{b}} \pm 0.03$ & $15.16^{\mathrm{ab}} \pm 0.24$ \\
\hline & Group C & $4.47^{\mathrm{a}} \pm 0.17$ & $0.46^{\mathrm{c}} \pm 0.02$ & $12.38^{\mathrm{c}} \pm 0.21$ \\
\hline \multirow{2}{*}{$\begin{array}{l}\text { Breed } \\
\text { (observation No. }=108 \\
\text { for each breed) }\end{array}$} & $\begin{array}{l}\text { White } \\
\text { Nicholas }\end{array}$ & $4.50^{\mathrm{a}} \pm 0.10$ & $0.98^{\mathrm{a}} \pm 0.04$ & $16.87^{\mathrm{a}} \pm 1.27$ \\
\hline & Black Baladi & $3.16^{\mathrm{b}} \pm 0.09$ & $0.64^{\mathrm{b}} \pm 0.03$ & $12.67^{\mathrm{b}} \pm 0.43$ \\
\hline \multirow{3}{*}{$\begin{array}{l}\text { Age } \\
\text { (observation No. }=72 \text { for } \\
\text { each week) }\end{array}$} & $18^{\text {th }}$ week & $3.73^{b} \pm 0.12$ & $0.76^{\mathrm{b}} \pm 0.05$ & $14.45^{\mathrm{a}} \pm 0.33$ \\
\hline & $22^{\text {nd }}$ week & $3.73^{\mathrm{b}} \pm 0.12$ & $0.76^{\mathrm{b}} \pm 0.05$ & $14.45^{\mathrm{a}} \pm 0.33$ \\
\hline & $24^{\text {th }}$ week & $4.02^{\mathrm{a}} \pm 0.18$ & $0.90^{\mathrm{a}} \pm 0.05$ & $15.41^{\mathrm{a}} \pm 0.12$ \\
\hline \multicolumn{5}{|c|}{ Breed X Heat stress (observation No. $=36$ for each treatment) } \\
\hline \multirow[t]{2}{*}{ Group A } & $\begin{array}{c}\text { White } \\
\text { Nicholas }\end{array}$ & $4.54^{\mathrm{b}} \pm 0.04$ & $1.52^{\mathrm{a}} \pm 0.05$ & $17.87^{\mathrm{a}} \pm 0.12$ \\
\hline & Black Baladi & $2.90^{\mathrm{d}} \pm 0.06$ & $0.82^{\mathrm{c}} \pm 0.04$ & $15.67^{\mathrm{a}} \pm 0.22$ \\
\hline \multirow[t]{2}{*}{ Group B } & $\begin{array}{l}\text { White } \\
\text { Nicholas }\end{array}$ & $3.70^{\mathrm{c}} \pm 0.18$ & $0.90^{\mathrm{b}} \pm 0.03$ & $16.07^{\mathrm{a}} \pm 0.11$ \\
\hline & Black Baladi & $2.91^{\mathrm{d}} \pm 0.05$ & $0.69^{\mathrm{d}} \pm 0.05$ & $14.25^{\mathrm{a}} \pm 0.42$ \\
\hline \multirow{2}{*}{ Group C } & $\begin{array}{l}\text { White } \\
\text { Nicholas }\end{array}$ & $5.25^{\mathrm{a}} \pm 0.15$ & $0.52^{\mathrm{e}} \pm 0.01$ & $16.67^{\mathrm{a}} \pm 3.83$ \\
\hline & Black Baladi & $3.70^{c} \pm 0.25$ & $0.40^{\mathrm{f}} \pm 0.03$ & $8.08^{\mathrm{b}} \pm 0.71$ \\
\hline
\end{tabular}

Differences between means within column having different superscripts arehighly statistically significant $(p \leq 0.01)$

$A=$ birds reared at $24{ }^{\circ} C \pm 2, B=$ birds exposed to heat stress at $30^{\circ} \mathrm{C} \pm 2, C=$ birds exposed to heat stress at $35^{\circ} \mathrm{C} \pm 2$

\section{Discussion}

\section{Productive performance parameters:}

Regarding the effect of heat stress on productive performance parameters, the decrease live body weight, body weight gain, and depressed food conversion ratio in heat stressed groups could be due to the decreased appetite and feed intake of bird, because feed energy must be used for muscle contraction associated with panting, at the same time high temperature decreased body weight gain and this effect was positively correlated with age (Daghir, 1995; Yahav et al, 1998; Yalcin et al, 1997). These results were in accordance with Monk (1998) and Brown (1997) who stated that heat-exposed birds will consume less feed in order to reduce the thermogenic effect associated with nutrient absorption, assimilation, and utilization. Decreased feed consumption and reduced blood flow to the gastrointestinal tract decreases metabolic energy input. These factors contribute to depressed 
performance characteristics of birds exposed to heat.

Heat stress showed a highly significant effect on food conversion ratio $(p \leq 0$. 01) this indicate that heat stress affects greatly FCR in turkey and this in agreement of Reece and Lott (1983) and Donkoh (1989) who stated that heat stress negatively affects feed efficiency in growing birds.

Breed effect and interaction between breed and heat stress revealed a great influence of White Nicholas breed on the productive performance parameters and this result could be attributed to a great difference in body weight of turkey breeds as the Black Baladi breed had a poor growth rate and a small body weight in comparison to the White Nicholas breed (Amin, 1999; EL-Naggar et al, 1992). Also, the obtained results were in agreement with Austic and Neshein (1990) results as they observed a slow growth and poor feed efficiency for small variety turkey and turkey performance is highly dependent upon environmental conditions, especially ambient temperatures. Furthermore, Brown (1997) and Monk (1998) stated that the interaction between more than one heat treatment $\left(30,35^{\circ} \mathrm{C}\right)$ with different groups of turkeys lead to increase in feed conversion ratio $1.7 \%$ per $1^{\circ} \mathrm{C}$ over a baseline of 18 $22^{\circ} \mathrm{C}$ in birds kept at 30 or $35^{\circ} \mathrm{C}$ as compared with those at lower temperatures.
The low feed intake, weight gain and fed conversion in a heat acclimated group are in accordance with the results reported by Van et al (1992). Meanwhile, Yahav and Hurwitz (1996) found a significant improvement in feed efficiency due to early heat stress. In general, body weight gain of turkeys will be retarded if their diet is not balanced properly for amino acid content or their environment is too hot, or both. The capacity of turkeys to dissipate heat to the environment reaches the upper limit in these circumstances (Ferket et al, 1995)

Age had a highly significant $(\mathrm{P} \leq 0$. 001) effect on turkey live body weight and this in accordance with Mostafa and Nofal (2000) and ElLaithy (2003) who observed that increasing age till 24 weeks old accompanied with an increase in weight $(\mathrm{P} \leq 0 . \quad 001)$. Also the findings of Yahav et al (1998) agreed with this result, they said that as with increasing of age the turkeys obtained an increase in body weight and body weight gain and exposing young turkeys during the first period of development to various environmental conditions may affect latter on their growth.

On the contrary Erosy et al (2006) and Parasad (2000) suggested that the best marketing age in turkeys is (16 weeks); maybe they mean the heavy breeds only, while Singh and Moore (1972) suggested selling turkeys at (20 weeks); also Mukesh (2005) reared turkeys till 24 weeks and he found that the profit per bird 
for both male and female was found maximum in 16 weeks of rearing, followed by 20 weeks and 24 weeks. Therefore, instead of waiting for highest body weight, it is better to sell turkey at 16 to 20 weeks of age to take maximum advantage of the higher weight gain, higher efficiency of feed utilization and higher profit.

But the obtained results had shown the profit per bird was at 16 weeks of rearing, followed by 20 weeks and was found maximum of 24 weeks, and the turkey farm owners in Egypt are rearing turkeys till (2021 weeks), so this decision when to sell turkeys depend upon many factors such as ration price, turkey meat price, season to take maximum advantage of the higher weight gain, higher efficiency of feed utilization and higher profit. Age effect was highly significant $(p \leq 0.001)$ on weight gain of bird indicating that turkeys obtained more than $80 \%$ of final body weight and final body weight gain in 24 weeks age (Mukesh, 2005, 2006 and El-Laithy, 2003).

Age had a high significant effect on turkey FCR at the period from 1416 and from 8-10 weeks, there was a great difference in body weight of turkey breeds and the Black Baladi breed had a poor growth rate and a small body weight in comparison with the White Nicholas breed (Amin, 1999 and EL-Naggar et al ,1992). Additionally, there was a significant increase in body weight was recorded up to 28 weeks in male and 20 weeks in female. Male turkey had significantly higher body weight than that of female in all ages. Feed efficiency was best at earlier age but up to 16 weeks (3.77) for male after that, it deteriorates progressively (Mukesh, 2006).

\section{Effects of heat stress on blood parameters and hormones:}

The result of RBC is in harmony with that of Khan et al (2002) who found that high ambient temperature decreases (TRBC) Total Red Blood Cells Count, which might be due to a decrease in oxygen consumption by turkeys, as a result of high ambient temperature, which might be associated with a concurrent reduction in the production of red blood cells (depressed hemopoetic activity) as a consequence of lower basal metabolic rate Aengwanich and Chinrasri (2002).

The decrease in hemoglobin concentration is in accordance with that reported by Aengwanich and Chinrasri (2002); Vecerek et al (2002); Khan et al (2002) and Comito et al (2007) who reported that the exposure of birds to high temperature cause significant decrease in hemoglobin concentration. Aengwanich (2007) indicated that heat stress decreases haemoglobin in all strains, there was significant $(\mathrm{P}<0.05)$ decrease during summer compared to winter seasons, which could be attributed to the decrease of salt content in blood plasma. In addition it could 
be justified by the reduction of RBC counts during the summer. The differences between strains in measured parameters can be related to the fact that a lot of variations exist among the different breeds of broiler chickens (Mmereole, 2009). Results of the experiment of differential leucocyte count represent significant increase in basophils $\%$ and lowered lymphocytes associated with high $\mathrm{H} / \mathrm{L}$ ratios showed the severe effect of heat stress (Ozkan et al, 2004) in both heat stressed groups (B and C) and this result could be attributed to the corticotropin- releasing hormone (CRH) which secreted by the paraventricular nucleus of the hypothalamus in response to heat stress stimulated adrenocorticotropic hormone $(\mathrm{ACTH})$ production from the anterior lobe of the pituitary gland in poultry. The increasing of blood corticosterone levels in the poultry under heat stress led to increase the concentration of circulating heterophils. As a result, heat stress causes an increase in the ratio of heterophil to Lymphocyte (H/L) due to the reduction of circulating lymphocytes and the higher numbers of heterophils in plasma of poultry (Lara and Rostagno, 2013 and Sahin et al, 2013). Meanwhile, it is mentioned that the $\mathrm{H} / \mathrm{L}$ ratio in blood is a more enduring and reliable indicator for the long-term stresses including heat stress than assessing corticosterone levels in poultry (Altan et al, 2000; Zulkifli et al, 2009; Kamboh et al, 2013). In addition, this result could be supported by result of increase in concentration of corticosteron hormone in group $\mathrm{C}$.

Breed effect on $\mathrm{WBC}$ and $\mathrm{H} / \mathrm{L}$ ratio revealed significant decrease in WBC and lymphocyte\% with significant increase in $\mathrm{H} / \mathrm{L}$ ratio in Black Baladi comparing with White breed and these results is in accordance with Eromomhene (2011) who found that the heterophils count is significant highest in black breed followed by white respectively so that, the black plumage color are more susceptible to stress, this discordant submission could be attributed to environmental and seasonal factors as heterophils count increases during stress as observed by Olayemi and Ojo (2007). At the same time, this indicates the presence of highly adverse environmental conditions which requires a highly resistive breed (white turkey breed) to survive. Also these results could explain the interaction between heat stress and breed where negative effect of heat stress was observed in black Baladi at highest heat stress $\left(35^{\circ} \mathrm{C} \pm 2\right.$ ).

Age effect had non-significant impact on in TWBC, this result was in agreement with the results of Elizabeth et al (2009), as they showed that there were no significant differences $(p>0.05)$ for hemoglobin, TWBC, heterophiles, monocytes and the $\mathrm{H} / \mathrm{L}$ ratio with age and gender. Also the recorded 
data was in complete harmony with Bounous et al (2000) results as they found that the total RBC and WBC count increase with age, also lymphocytes and basophiles differs greatly with increasing age.

The increased thyroxine level could be used as a criterion for significant worsening of the poultry welfare (Bozakova et al, 2007).

The thyroid secretion rate had actually decreased at the higher temperature (Falconer, 1984). The decrease of $\mathrm{T} 3$ and $\mathrm{T} 4$ levels in heat stressed group $\mathrm{C}$ which associated with the significant increase in corticosterone are most likely related to the adaptation to changes in metabolic demands caused by physiological stress (Davis et al, 2000). Also Triiodothyronine (T3) is hormones known to effect growth rate and food intake which is the main metabolic hormone (Yahav, 2000).

An increase in $\mathrm{T} 3$ and $\mathrm{T} 4$ in elder age might be due to more adaptation to the elder birds whereas; the importance of thyroid gland hormones in adaptation to heat stress is related to the central role that thyroid hormones play in the regulation of metabolic rate of birds (McNabb, 1988). Also this phenomenon could be explained by the adaptation of birds to changes in metabolic demands caused by physiological stress and increased metabolic activity with the age (Melesse et al, 2011).

\section{Conclusion}

The above results indicate that exposure of birds to high temperature ( 30 and $35^{\circ} \mathrm{C} \pm 2$ ) lead to depression of productive performance parameters with altering TRBC, TWBC and increasing $\mathrm{H} / \mathrm{L}$ ratio and corticosterone concentration, also alter body temperature regulating thyroid hormones. In addition, the breed has great influence on the above measured parameters. Heat stress and breed interaction revealed that Black Baladi breed which reared at higher temperature $\left(35^{\circ} \mathrm{C} \pm 2\right)$ is more sensitive to the heat stress than White Nicholas. Therefore, it is necessary to keep birds at comfortable environmental temperature to obtain maximum production and immunity and to maintain the balance thermoregulation system; at the same time, it is important in production system to choose breed can tolerate the possible heat increase due to any environmental and climatic change.

\section{References}

Altan, O., Altan, A., Cabuk, M., Bayraktar, H., (2000): Effects of heat stress on some blood parameters in broilers. Turk. J. Vet. Anim. Sci. 24:145-148. 
Aengwanich, $W$ and Chinrasri, $O$ (2002): Effect of heat stress on body temperature and hematological parameters in male layers. Thai J. Physiol. Sci., 15: 2733.

Austic, R.E and Neshein, M.C. (1990): Poultry production 13th ed.Lea and Febiger, Philadelphia, P. A. K. M. Varghese Company, Bombay, India. 6-22 pp.

Amin, E.M. (1999): Effects of crossbreeding and feeding different levels of Atriplex meal on production characters of local and commercial varieties of turkey, M.Sc. Thesis.Fac.of Agric., Alex.Unive., Egypt.

Bounous, D.I.; Wyatt, R.D.; Gibbs, P.S.; Kilburn J.V. and Quist, C.F. (2000): Normal hematologic and serum biochemical reference intervals for juvenile wild turkeys. J. Wildlife Dis., 36: 393396.

Bozakova, N.; Popova ,S. and Ralcheva (2007): Thyroid hormone level and relative liver weight in male turkeys in relationship with their welfare, Biotechnology in Animal Husbandry 23 (5-6), p 511 518 , 2007, Institute of Animal Science Kostinbrod, Bulgaria.

Brody, S. (1968): Bioenergetics and growth .Hafher publ.Inc.N.Y.

Brown, R. H. (1997): Heat wave reduces broiler, turkey population in Southeast. Feedstuffs 58:34:10.

Christie, (1978): Clinical Chemistry, The theory of reference values. International Federation of
Clinical Chemistry. Acta 87: 459F475F.

Comito, R. W.; Reece W. O.; Trampel D. W. and Koehler K. J. (2007):

Physiology, Endocrinology, and Reproduction Acid-Base Balance of the Domestic Turkey during thermal panting. College of Veterinary Medicine; Iowa State University, Ames 50011 2007 Poultry Science 86:26492652.

Daghir, N.J. (1995): Nutrient requirements of poultry at high temperature. pages 101123: Poultry Production in Hot Climates. N. J. Daghir, CAB International, University, Cambridge, U.K.

Davis, G.S.; Anderson, K.E.; Carrol, A.S. (2000): The effects of long term caging and molt of Single Comb White Leghorn hens on heterophil to lymphocyte ratios, corticosterone and thyroid hormones. Poult Sci., 2000 Apr.; 79 (4):514-8.

Deeb, N. and Cahaner, A. (2002): Genotype-by-environment interaction with broiler genotypes differing in growth rate. 3 . Growth rate and water consumption of broiler progeny from weightselected versus nonselected parents under normal and high ambient temperatures. Poult. Sci. 81, 293301.

Donkoh, A. (1989): Ambient temperature a factor affecting performance and physiological response of broiler chickens. International J. Biometeorology 33: 259-265. 
El-Laithy S. M. Moursy (2003): Effect of some managerial factors on turkey performance and behaviour, Ph.D.Thesis, faculty of veterinary medicine, department of animal hygiene, behaviour, and management, Moshtohor, Banha university. Pp: 1-175

El-Naggar, N. M., Sharaf, M.M and Mandour, M.A. (1992): Relationship of carcass yields and some body measurements in different turkey strains. Egypt. Poultry Science, 12: 145-164.

Elnagar, S.A.; Scheideler, S.E.; Beck, M.M. (2010): Reproductive hormones, hepatic deiodinase messenger ribonucleic acid, and vasoactive intestinal polypeptideimmunoreactive cells in hypothalamus in the heat stressinduced or chemically induced hypothyroid laying hen. Poult. Sci., 89, 2001-2009.

Elizabeth, M. d. S. S.; Paulillo, A. C.; Martins, G. R. V.; Ivan M. L., Alan J. P. T., L. N. J. J. D. and José J. F. (2009): Hematology of the Bronze Turkey (Meleagris Gallopavo): Variations with Age and Gender. International Journal of Poultry Science 8 (8): pages 752754, 2009.

Eromomhene, A. (2011): Effects of color variants on the differential of white blood cell and packed cell volume concentration in Nigerian local turkey. A Project Report Submitted to The Department of Animal Breeding and Genetics, College of Animal Science and
Livestock Production. University of Agriculture, Abeokuta.

Erosy, I. E.; Mendes M. and Sedat A. (2006): Growth curve establishment for American Bronze turkeys. Arch. Tierz., Dummerstorf 49 (2006) 3, 293-299.

Euribrid, B. V. (1982): Technical Information for Hybro Broilers, P.22 (Boxmeer, the Netherslands, Euribrid poultry breeding farms).

Falconer, I. R. (1984): the thyroid glands.In:'Physiology and biochemistry of the domestic fowel. Vol 5. Chapter 4.

Ferket, P. R.; Grimes J. L.; J. Brake, and D. V. Rives (1995): Effects of dietary virginiamycin, arginine: lysine ratio, and electrolyte balance on the performance and carcass yield of turkey toms. Poultry Science (supplement 1): 74-190.

Garriga, C.; Hunter, R.R.; Amat, C.; Planas, J.M.; Mitchell, M.A.; Moreto, M. (2006): Heat stress increases apical glucose transport in the chicken jejunum. Am. J. Physiol. Reg. Integ. Comp. Physiol. 2006, 290, R195-R201.

Gross, W.B. and Siegel, H.S., (1983): Evaluation of the heterophil/lymphocyte ratio as a measure of stress in chickens. Avian Diseases. 27: 972-979.

Gu, X.H., Hao, Y., Wang, X.L., (2012): Overexpression of heat shock protein 70 and its relationship to intestine under acute heat stress in broilers: 2. Intestinal oxidative stress. Poultry Sci. 91:790-799 
Kamboh, A.A., Hang, S.Q., Bakhetgul, M., Zhu, W.Y., (2013): Effects of genistein and hesperidin on biomarkers of heat stress in broilers under persistent summer stress. Poultry Sci. 92:2411-2418.

Khan, W.A., Khan A. (2002): Pathological effects of induced heat stress in broilers chicks. Pakistan, Vet. J., 13-100.

Lara, L.J., Rostagno, M.H., (2013): Impact of heat stress on poultry production. Animal 3:356369.

Mack, L.A.; Felver-Gant, J.N.; Dennis, R.L.; Cheng, H.W. (2013): Genetic variations alter production and behavioral responses following heat stress in two strains of laying hens. Poult. Sci. 2013, 92, 285-294.

Maxwell, M. H.; Robertson, G. M.; Mitchell, M.A. and Carlisle A. J. (1992): The fine structure of broiler chicken blood cells, with particular reference to basophils, after severe heat stress. Comparative Haemotology International. 1992a; 2:190-200.

McKee S. R. and Sams A. R. (1997): The effect of seasonal heat stress on rigor development and the incidence of pale, exudative turkey meat. Poult. Sci. 76:1616-1620.

McNabb F.M.A. (1988):
Peripheral thyroid hormone
dynamics in precocial and altricial
avian development. Am. Zool., 28:
427-440.

Melesse, A., Maak ,S. Schmidt, R. and Lengerken, G. von (2011): Effect of long-term heat stress on key enzyme activities and T3 levels in commercial layer hens. International Journal of Livestock Production Vol. 2(7), pp. 107-116.

Mercia, L. (1986): Raising Poultry the modern way.Sixteenth Printing.P:108.

Mmereole, F.U.C (2009): Effects of age and breeds on the haematological parameters of broilers Natural and Applied Sciences Journal, 10(1), 90-95.

Monk, A. (1998): Effects of heat stress as a cascade problem, poultry welfare. 1998, pp: 35-72.

Mostafa, M. Y. and R. Y. Nofal (2000): Effects of crossing two breeds of turkeys on libe bodies' measurements, growth performance and livability. Egyptian Poultry Sci. Vol. 20(II) June (239-252).

Mukesh Karki (2005): Growth, Efficiency of Feed Utilization and Economics of Different Rearing Periods of Turkeys, Regional Agricultural Research StationNARC, Parwanipur, PO Box 4, Nepal Agric. Res. J. Vol. 6, pp:8488.

Mukesh Karki (2006): Economics of Different Rearing Periods of Turkeys, Regional Agricultural Research Station- PO Box 4, Nepal Agric. Res. J. Vol. 6, pp:84-88

National Research Council (1994): Nutrient Requirements of poultry $.9^{\text {th }}$ revised edition. National Academy Press .Washington.D.C.

Olayemi, F.O. and O.E. Ojo, (2007): Haematology of the Nigerian turkey .Folia Vet., 51: 4346. 
Osman A. M. K., E. S., Tawfic F. W., Klien, and W. Hebeler (1989): Effect of environmental temperature on growth, carcass traits and meat quality of broilers of both sexes and different ages.53: 163175.

Özkan, S., Çabuk, M. and Konca, Y. (2004): Leukocyte Responses to Acute Heat Stress in Turkey Toms Either Fed Restricted or Ad Libitum During Growth Period. XXII. World's Poultry Congress, İstanbul. Prasad D. (2000): Poultry farming. 2nd edition. Kalyani Publishers, New Delhi, India.

Quinteiro-Filho, W.M.; Gomes, A.V.; Pinheiro, M.L.; Ribeiro, A.; Ferraz-de-Paula, V.; AstolfiFerreira, C.S.; Ferreira, A.J.; Palermo-Neto, J.(2012): Heat stress impairs performance andinduces intestinal inflammation in broiler chickens infected with Salmonella Enteritidis. Avian Pathol. 2012, 41, 421-427.

Reece, F.N., and B.D. Lott (1983): Typical broiler chicken growth rates Poultry Sci., 61: 1013-1015.

Richards F. J. (1959): A flexible growth functions for empirical use. J. Exp. Bot. 10 (1959), 290-300.

Sahin, K., Orhan, C., Smith, M.O., Sahin, N., (2013): Molecular targets of dietary phytochemicals for the alleviation of heat stress in poultry. World Poultry Sci. J. 69:113-123.

Sainsbury D.W. (2000): Poultry health and management. 4th edition. P: 151-152.

Scott, T. A., and D. Balnave (1988): Comparison between concentrated complete diets and self-selection for feeding sexually maturing pullets at hot and cold temperatures. Br. Poult. Sci. 29:613-625.

Škrbić Z., Pavlovski, M. Lukić (2009): Stocking Density - factor of production performance, quality and broiler welfare, Biotechnology in Animal Husbandry 25 (5-6), p 359-372, 2009, Publisher: Institute for Animal Husbandry, BelgradeZemun ISSN 1450-9156

Singh $H$ and EN Moore (1972): Livestock and poultry production. 2nd edition. Prentice Hall of India Private Limited, New Delhi, India.

Star, L.; Decuypere, E.; Parmentier, H.K.; Kemp, B. (2008): Effect of single or combined climatic and hygienic stress in four layer lines: Endocrine and oxidative stress responses. Poult. Sci., 87, 1031-1038.

Star, L.; Juul-Madsen, H.R.; Decuypere, E.; Nieuwland, M.G.; de Vries Reilingh, G.; van den Brand, H.; Kemp, B.; Parmentier, H.K. (2009): Effect of early life thermal conditioning and immune challenge on thermotolerance and humoral immune competence in adult laying hens. Poult. Sci., 88, 2253-2261.

Van Der Hel, W.; Vestegen, M.W.A.; Pijls, L. and van Kampen, M. (1992): Effect of twoday temperature exposure of meonatal broiler chicks on growth performance and body composition during two weeks at normal 
condition. Poult. Sci. 71: 20142021.

Večerek, E. Strakovă; Suchŷ P. and Voslářová, E. (2002): Influence of high environmental temperature on production and haematological and biochemical indexes in broiler chickens, Czech J. Anim. Sci., 47, 2002 (5): 176-182.

Waibel, P.E.; Carlson, CW; Brannon J.A. and Noll, S.L. (2000): Limiting amino acids after methionine and lysine with growing turkeys fed low-protein diets. Poultry 79:1290-1298.

Yahav, S. (2000): Relative humidity at moderate ambient temperatures: its effect on male broiler chickens and turkey.British Poult. Sci., 41: 94-100.

Yahav, S. and Hurwitz, S. (1996): Induction of thermotolerance in male broiler chickens by temperature conditioning at an early age, Poult. Science. 75: 402-406.

Yahav, S.; Plavnik, I.; Rusal, M. and Hurwitz, S. (1998): Response of turkey to relative humidity at high ambient temperature. British poultry science, 39:340-345.

Yalcin S.; Testik A.; Ozkan S., Celen F., and Cahaner, A. (1997): Performance of naked neck and normal broilers in hot, warm and temperature climates. Poultry Sci., 76: 930-937.

Zulkifli, I., Al-Aqil, A., Omar, A.R., Sazili, A.Q., Rajion, M.A., (2009): Crating and heat stress influence blood parameters and heat shock protein 70 expression in broiler chickens showing short or long tonic immobility reactions. Poultry Sci. 88:471-476. 


\section{الملخص العربى}

\section{دراسة تاثير الاجهاد الحرارى على الاداء الانتاجى وقياسات الام لسلالات الرومى الإسى

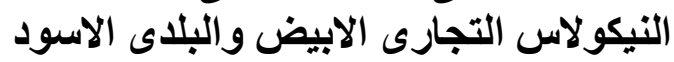

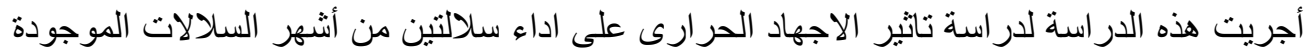

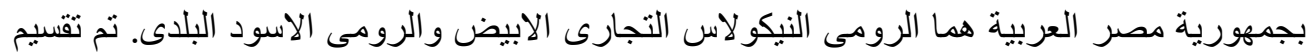

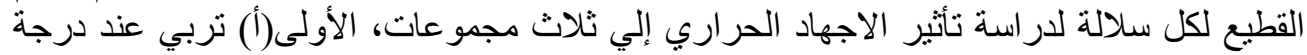

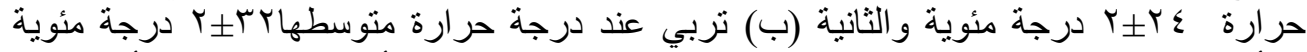

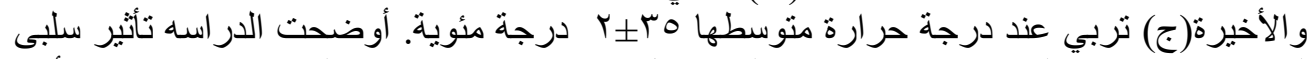

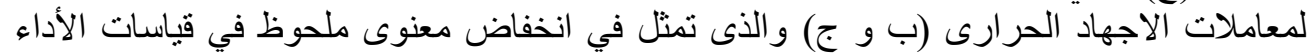

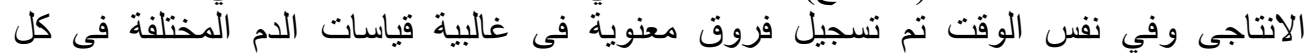

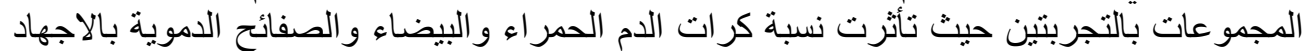

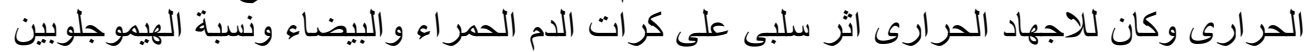

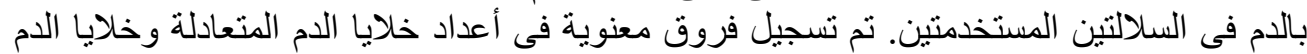

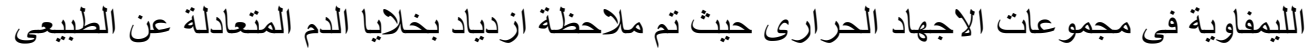

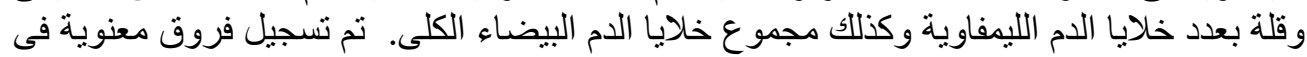

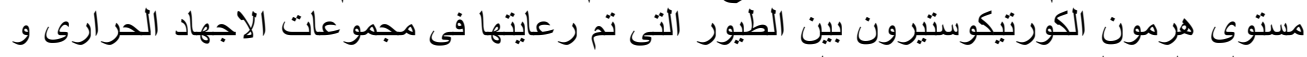

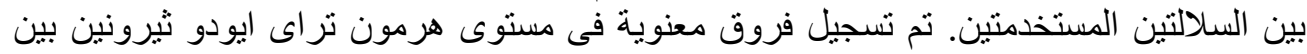

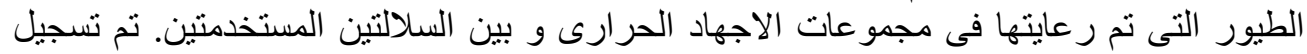

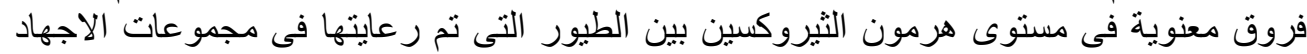

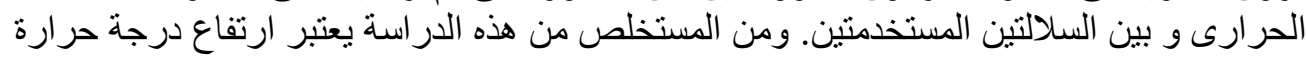

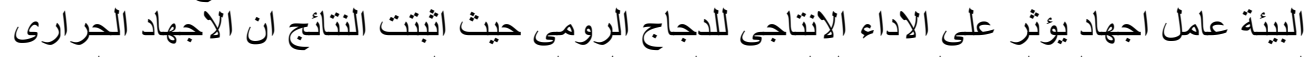

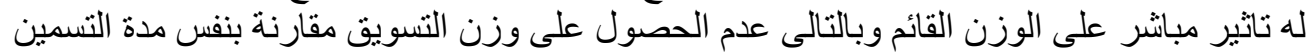

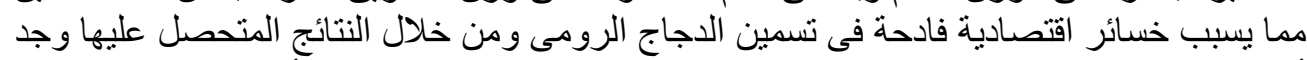

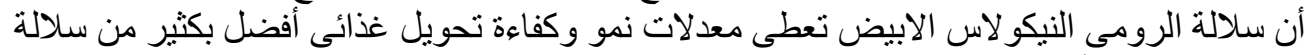

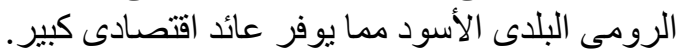

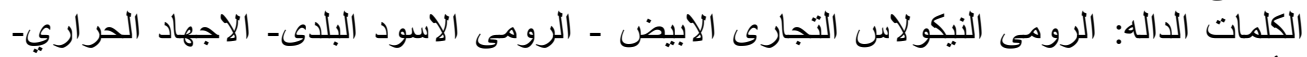

